\title{
Through the kaleidoscope: star formation the host galaxies of radio-AGN
}

\author{
Marios Karouzos ${ }^{1}$, Myungshin Im $^{1}$, Markos Trichas ${ }^{2}$, Tomo Goto ${ }^{3}$, \\ Matt Malkan ${ }^{4}$, Angel Ruiz ${ }^{5}$, Yiseul Jeon ${ }^{1}$, and the AKARI NEP team \\ ${ }^{1}$ CEOU - Astronomy Program, Department of Physics \& Astronomy, \\ Seoul National University, Gwanak-gu, Seoul, Korea \\ email:mkarouzos@astro.snu.ac.kr \\ ${ }^{2}$ EADS Astrium Ltd., Gunnels Wood Road, Stevenage SG1 2AS, UK \\ ${ }^{3}$ Dark Cosmology Centre, Niels Bohr Institute University of Copenhagen, \\ Juliane Maries Vej 30, 2100 Copenhagen, Denmark \\ ${ }^{4}$ Division of Astronomy and Astrophysics, 3-714 UCLA, LA CA 90005-1547, USA \\ ${ }^{5}$ Inter-University Centre for Astronomy and Astrophysics (IUCAA), \\ Post Bag 4, Ganeshkhind, 411077 Pune, India
}

\begin{abstract}
There exist strong evidence supporting the co-evolution of central supermassive black holes and their host galaxies; however it is still under debate how such a relation comes about and whether it is relevant for all or only a subset of galaxies. A rich multi-wavelength dataset is available for the North Ecliptic Pole field, most notably surveyed by the AKARI infrared space telescope. We investigate the star-formation properties of the host galaxies of radio-AGN together with the radio feedback mechanism, potentially responsible for the eventual quenching of star formation. Using broadband SED modelling, the nuclear and host galaxy components of these sources are studied as a function of their radio luminosity. Here we present results concerning the AGN content of the radio sources in this field, while offering evidence supporting a "maintenance" type of feedback from powerful radio-jets.
\end{abstract}

Keywords. galaxies: active, galaxies: evolution, galaxies: jets, radio continuum: galaxies, infrared: galaxies

Feedback from an accreting supermassive black hole (SMBH; i.e., the deposition of some of the accretion energy into the surrounding medium) is a physical process that has been suggested as one of the processes that connects the growth of SMBHs and their host galaxies (e.g., Fabian 2012). Feedback comprises a large number of potentially different mechanisms that affect and effectively regulate the growth of either the host galaxy, or the central SMBH, or both. Feedback has been shown to be important in large cosmological simulations (e.g., Croton et al. 2006). The regulated growth of SMBHs and their host galaxies has been labelled as the co-evolution scenario (for a review of this, see Kormendy \& Ho 2013).

Here we focus on the feedback from radio-loud AGN, in particular on the impact that their collimated outflows, radio-jets, have on their host galaxies. These radio-jets are able to deposit large quantities of mechanical energy in their surroundings (e.g., McNamara et al. 2005). Results concerning the role of radio-loud AGN in the evolution of galaxies, in particular with respect to star formation $(\mathrm{SF})$, can be divided in two broad families, one advocating for radio-jets effectively inhibiting global star SF (e.g., Morganti et al. 2013), with the other supporting a positive SF feedback (e.g., Gaibler et al. 2012).

Although some form of feedback appears to be necessary to explain the results supporting the common growth of central spheroids and their galaxies, the exact nature of such a feedback remains unclear. We therefore investigate SF in the host galaxies of 
radio-AGN in order to assess whether and to what extent radio-jets can inhibit SF in their host galaxies. To do this, we investigate the broadband spectral energy distributions (SEDs) of a sample of radio sources and decouple the AGN and SF components.

\section{Data and Method}

We use data from the AKARI infrared space telescope (Murakami et al. 2007) survey of the North Ecliptic Pole (NEP) field, covering the wavelength range from 2 to 24 microns. Ancillary multi-wavelength data come from the Westerbork Synthesis Radio Telescope (WSRT) at $1.4 \mathrm{GHz}$ (White et al. 2010), from the Hershel-SPIRE instrument in the far-IR (Pearson et al., in prep.), from the CFHT in the optical and near-IR (Oi et al. 2013, subm.), and from GALEX in the UV (Malkan, private communication). In total, we cross-match 321 radio-sources at $1.4 \mathrm{GHz}$ (at a $\sim 0.1 \mathrm{mJy}^{\text {beam }}{ }^{-1}$ detection limit) with AKARI sources detected at $5 \sigma$ in the NIR (see Karouzos et al. 2014a).

For these sources we model the optical to NIR SEDs to calculate photometric redshifts using the publicly available photometric redshift code LePhare (Arnouts et al. 1999, Ilbert et al. 2006). Due to the partial coverage of full-band optical data, 237 out of 321 sources end up with a reliable photometric and/or spectroscopic redshift. We focus our analysis on these sources.

For the modelling of the full broadband SEDs of these 237 sources we employ a template-fitting method developed for Ruiz et al. (2010) and Trichas et al. (2012). The optical/NIR SED (wavelengths shortward of $3.2 \mathrm{um}$ ) and the IR SED are fitted separately using two sets of nine and four templates (AGN, SF, and passive), respectively, using a $\chi^{2}$ minimization technique. Finally, the optical and IR best-fit models were added to obtain a complete model of the SED covering the entire wavelength range.

From the fit we can constrain the IR luminosity of both the SF and AGN components. Moreover, we can calculate the total contribution of the AGN to the total SED luminosity, the SF rate (SFR; inferred by the total IR luminosity of the SF component), the stellar mass of the host galaxy (from the rest-frame 2.3 micron luminosity), and its specific SFR (SFR per unit mass).

\section{Characterising the population of radio sources}

At the sub-mJy depth of the $1.4 \mathrm{GHz}$ WSRT survey of the NEP, the selected sample should be dominated by star-forming galaxies, whose supernovae population produces the radio emission that puts them above our radio flux density selection limit. Alternatively, galaxies that harbour an AGN with a radio-jet would also be picked up by the radio selection. It is therefore important to constrain the AGN content of the sample.

We first look at the contribution of $\mathrm{AGN}$ as a function of the radio luminosity in Fig. 1 (left). We find an excess of AGN-composite or AGN-dominated sources at intermediate and high radio luminosities. We also see that the rise in the AGN contribution in terms of bolometric luminosity agrees well with previous studies for the local Universe and it starts roughly at around $10^{40} \mathrm{erg} \mathrm{s}^{-1}$ (e.g., Mauch \& Sadler 2007). However, we also see significant scatter, with AGN identified at the lowest radio luminosities (presumably radio-quiet $\mathrm{AGN}$ ) as well as non-AGN found in the highest radio-luminosities (presumably vigorously star-forming galaxies). This drives the point that at the faintest radio flux densities the fraction of AGN is comparably smaller to that found in the bright radio surveys (like the FIRST and the NVSS), while the radio population is mainly dominated by star-forming (e.g., Seymour et al. 2004) or potentially composite galaxies (e.g, Strazzullo et al. 2010). This is corroborated by the WISE mid-IR colours of the sample, shown in the right panel of Fig. 1. Most of the sources cover the whole range of WISE colors for spiral galaxies, with a significant fraction falling within the starburst region. 

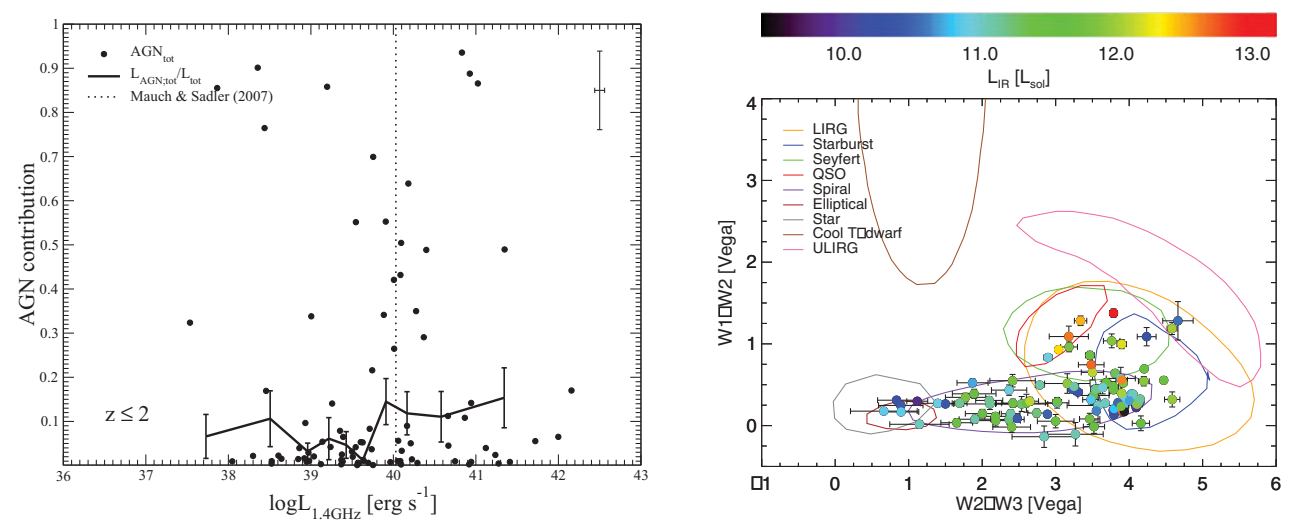

Figure 1. Left: The fractional contribution of the AGN component to the bolometric luminosity as derived from the SED fits as a function of total radio luminosity at $1.4 \mathrm{GHz}$. Points denote source values while lines show averaged values over radio luminosity bins. The total AGN contribution (optical + IR; filled circles) is plotted. The vertical black dashed line shows the rough luminosity cut where the luminosity function of local radio-sources gets dominated by AGN systems (from Mauch \& Sadler 2007). Right: W1-W2 color as a function of W2-W3 color for sources detected in all three WISE bands. The color-coding shows the total IR luminosity from the SED fitting. We adopt the same WISE color axis ranges for direct comparison to Fig. 12 of Wright et al. (2010). We only plot sources with $z \leqslant 2$.

Our sources do not exhibit strong red W1-W2 colors, indicating the absence of heavily extinct objects or typical Type 1 AGN (e.g., Stern et al. 2012).

\section{Star formation suppression by radio-jets?}

In Fig. 2 we plot the SFR as a function of radio luminosity for our sample (left) and the specific SFR as a function of redshift and radio luminosity (right). Focusing on the left panel first, we observe that at low radio luminosities, the sources follow the expected relation from Bell (2003) quite well, confirming that the radio emission in the majority of these sources is dominated by their SF. However, as we approach radio luminosities characteristic of radio-loud AGN (dashed line) we observe more source deviating from the expected relation, showing excess radio emission, most plausibly originating in a radio-jet. Above a radio luminosity of $\sim 10^{40} \mathrm{erg} \mathrm{s}^{-1}$, all sources fall well off the Bell relation. We note that in an absolute sense, these radio-loud/radio-excess sources still exhibit similar or higher SFRs than their lower radio-luminosity counterparts.

Turning to the right panel of Fig. 2, we now put our sample on the "Main Sequence" (MS) of SF, as defined by Elbaz et al. (2011). We calculate average specific SFR within radio luminosity bins. We observe that above the radio luminosity limit of $\sim 10^{40} \mathrm{erg}$ $\mathrm{s}^{-1}$ increased radio luminosity leads to a decrease in the specific SFR. The most radioloud/radio-luminous sources (shown in dark red in the plot) are found at the lower end of the MS, being consistent with normal SF galaxies at that redshift.

\section{Conclusions}

We find support for negative feedback in the host galaxies of radio-luminous AGN. Sources with the strongest radio-jets exhibit specific SFR consistent with very low starforming galaxies, at the lower end of the MS at their respective redshift. This is in perfect agreement with theoretical expectation of the "maintenance" nature of "radio-mode" feedback as opposed to the more energetic "QSO-mode" responsible for the putative transformation of sources into the red sequence. Future observatories like SPICA and the 

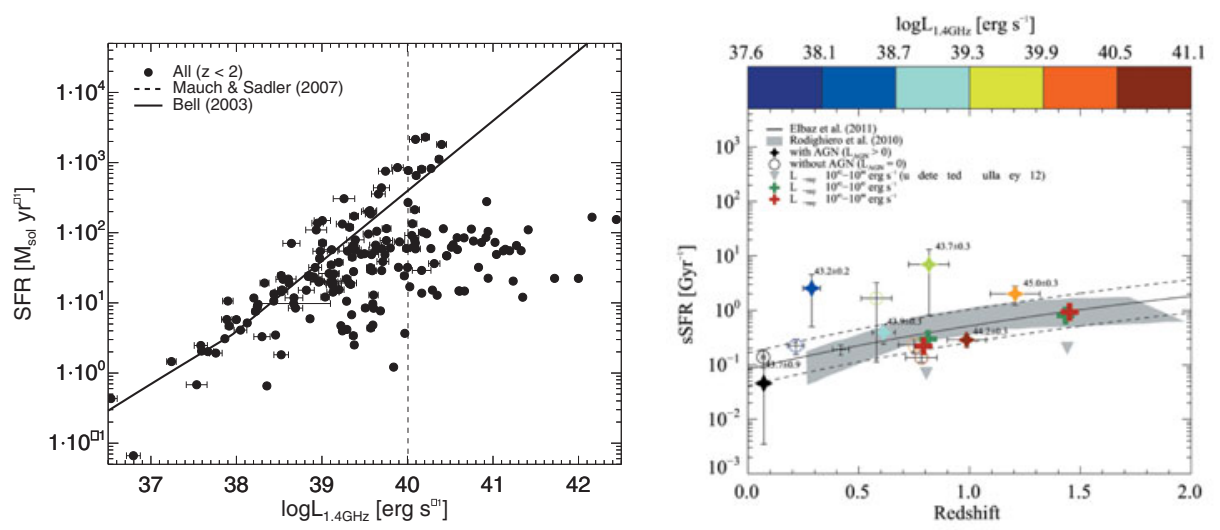

Figure 2. Left: Star-formation rate as a function of radio-luminosity at $1.4 \mathrm{GHz}\left(\mathrm{L}_{1.4 G H z}\right)$. The solid line denotes the relation between SFR and radio emission from supernovae taken from Bell (2003). The vertical dashed lines marks the radio-luminosity limit above which sources are usually classified as radio-AGN. Right: Specific SFR as a function of redshift for the AKARI-WSRT sources. The solid line shows the main sequence of star-forming galaxies as defined by Elbaz et al. (2011). We separate sources with an AGN component in their SED fit (filled stars) from sources with no AGN component (open circles). Next to each star the average value of the AGN-component luminosity within that radio-luminosity bin is given.

James Webb Space Telescope will reach much higher sensitivity and wider wavelength range and will therefore extend this study to both the fainter end of the optical luminosity function as well as the less massive end of the mass function of galaxies.

\section{References}

Arnouts, S., Cristiani, S., Moscardini, L., et al. 1999, MNRAS, 310, 540

Bell, E. F. 2003, ApJ, 586, 794

Bicknell, G. V., Sutherland, R. S., van Breugel, W. J. M., et al. 2000, ApJ, 540, 678

Croton, D. J., Springel, V., White, S. D. M., et al. 2006, MNRAS, 365, 11

Karouzos, M., Im, M., Trichas, M., et al. 2014a, ApJ, 784, 137

Elbaz, D., Dickinson, M., Hwang, H. S., et al. 2011, A\&3A, 533, A119

Fabian, A. C. 2012, Ann. Rev. Astron. Astrophys., 50, 455

Gaibler, V., Khochfar, S., Krause, M., \& Silk, J. 2012, MNRAS, 425, 438

Hwang, N., Lee, M. G., Lee, H. M., et al. 2007, ApJS, 172, 583

Ilbert, O., Arnouts, S., McCracken, H. J., et al. 2006, $A \& A$ A, 457, 841

Kormendy, J. \& Ho, L. C. 2013, Ann. Rev. Astron. Astrophys., 51, 511

Mauch, T. \& Sadler, E. M. 2007, MNRAS, 375, 931

McNamara, B. R., Nulsen, P. E. J., Wise, M. W., et al. 2005, Nature, 433, 45

Morganti, R., Frieswijk, W., Oonk, et al. 2013, A\&GA, 552, L4

Murakami, H., Baba, H., Barthel, P., et al. 2007, PASJ, 59, 369

Rowan-Robinson, M., Babbedge, T., Oliver, S., et al. 2008, MNRAS, 386, 697

Ruiz, A., Miniutti, G., Panessa, F., \& Carrera, F. J. 2010, A\&A, 515, A99

Seymour, N., McHardy, I. M., \& Gunn, K. F. 2004, MNRAS, 352, 131

Stern, D., Assef, R. J., Benford, D. J., et al. 2012, ApJ, 753, 30

Strazzullo, V., Pannella, M., Owen, F. N., et al. 2010, ApJ, 714, 1305

Trichas, M., Green, P. J., Silverman, J. D., et al. 2012, ApJS, 200, 17

Wagner, A. Y. \& Bicknell, G. V. 2011, ApJ, 728, 29

White, G. J., Pearson, C., Braun, R., et al. 2010, A\& A, 517, A54

Wright, E. L., Eisenhardt, P. R. M., Mainzer, A. K., et al. 2010, AJ, 140, 1868 